\title{
Long Noncoding RNA CCDC26 Promotes Thyroid Cancer Malignant Progression via miR-422a/EZH2/Sirt6 Axis
}

\author{
Xiao Ma' \\ Yanyan $\mathrm{Li}^{2}$ \\ Yuntao Song' \\ Guohui Xu' \\ 'Key Laboratory of Carcinogenesis and \\ Translational Research, Department of \\ Head and Neck, Peking University \\ Cancer Hospital and Institute, Beijing, \\ I00142, People's Republic of China; \\ ${ }^{2}$ Department of Cardiology, Air Force \\ Medical Center, Beijing, 100036, People's \\ Republic of China
}

Correspondence: Xiao Ma

Key Laboratory of Carcinogenesis and Translational Research, Department of Head and Neck, Perking University Cancer Hospital and Institute, No. 52 Fucheng Road, Haidian District, Beijing, I00I42, People's Republic of China Email XiaoMaResearch@163.com
Purpose: Long noncoding RNAs are crucial regulators in thyroid cancer progression. However, the role of IncRNA CCDC26 in thyroid cancer remains unclear. Here, we aimed to explore the effect of $\mathrm{CCDC} 26$ on thyroid cancer progression and the underlying mechanism.

Materials and Methods: A total of 50 clinical thyroid cancer samples were studied in patients' samples, cultured cells, and nude mice before and after treatment using quantitative reverse transcription-PCR, CCK-8 assays, BrdU incorporation assays, Transwell assays, cell apoptosis analysis, luciferase reporter gene assay, RNA immunoprecipitation, Western blot analysis, and tumorigenicity analysis.

Results: CCDC26 expression was elevated in patients' thyroid cancer tissues and thyroid cancer cell lines. CCDC26 depletion remarkably reduced proliferation, invasion, and migration but induced apoptosis of thyroid cancer cells. Mechanically, miR-422a mimic remarkably reduced the luciferase activity of CCDC26 transfected cells but failed to affect cells transfected with CCDC26 containing the mutated miR-422a-binding site. RNA immunoprecipitation (RIP) assays showed that CCDC26 and miR-422a preferentially interacted with Ago2, but not IgG, in the micro-ribonucleoprotein complexes (miRNPs). CCDC26 depletion enhanced miR-422a expression and MiR-422a inhibitor reversed CCDC26 knockdowninduced inhibition of thyroid cancer progression in vitro. CCDC26 upregulated EZH2 and Sirt6 expression by sponging miR-422a in thyroid cancer cells. Tumorigenicity analysis in nude mice revealed that CCDC26 contributed to thyroid tumor growth via miR-422a/EZH2/ Sirt6 axis in vivo.

Conclusion: CCDC26 promotes thyroid cancer malignant progression via miR-422a/EZH2/ Sirt6 axis. This finding provides new insights into the mechanism by which CCDC26 promotes malignant thyroid cancer development, advances our understanding of lncRNAs' association with thyroid cancer, and indicates that CCDC26 and miR-422a may serve as potential targets for thyroid cancer.

Keywords: thyroid cancer, CCDC26, miR-422a, EZH2, Sirt6

\section{Introduction}

Thyroid cancer is a common endocrine tumor with severe morbidity ${ }^{1}$ and steadily growing incidence, ${ }^{3,4}$ resulting in approximately $1 \%$ of cancer-related morbidity globally. $^{2}$ Although thyroid cancer displays a favorable prognosis, ${ }^{5}$ patients with aggressive metastasis exhibit considerably poor outcomes. ${ }^{6}$ Understanding the molecular mechanisms underlying thyroid cancer progression will benefit thyroid cancer diagnosis, therapy, and prognosis ${ }^{7,8}$ and is crucial and urgently required. 
However, the advancement in the mechanism investigation of thyroid cancer pathogenesis is still limited.

Long noncoding RNAs (lncRNAs), a type of noncoding RNAs of length $>200$ nucleotides, display fundamental functions in carcinogenesis. ${ }^{9-11}$ Many lncRNAs participate in thyroid cancer modulation. For example, lncRNA FOXD2-AS1 acts as a competing endogenous factor in thyroid cancer to modulate TERT expression by targeting miR7-5p. ${ }^{12}$ LncRNA TNRC6C-AS1 as a competing endogenous RNA mediates UNC5B to induce cell invasion, migration, and proliferation by modulating miR-129-5p in thyroid cancer. ${ }^{13}$ LncRNA DARS-AS1 targets microRNA-129 to increase thyroid cancer progression. ${ }^{14}$ Moreover, lncRNA CCDC26, as a poorly investigated lncRNA, plays crucial roles in several cancer models, such as pancreatic cancer, leukemia, and laryngeal squamous cell carcinoma. ${ }^{15-17}$ However, the effect of CCDC26 on the progression of thyroid cancer remains unclear.

MicroRNAs (miRNAs) are short noncoding RNAs with a length of approximately 20-25 nucleotides and have significant impacts on numerous biological processes, such as cell apoptosis, proliferation, differentiation, invasion, metastasis, and tumorigenesis by interacting with lncRNAs. ${ }^{18,19}$ MiRNAs post-transcriptionally control the expression of genes within the target mRNAs' $3^{\prime}$ untranslated region (3' UTR) ${ }^{20}$ Many investigations have revealed that miRNAs are involved in thyroid cancer progression. ${ }^{21}$ For instance, miR597-3p represses thyroid cancer cell invasion and migration by regulating $\mathrm{RAB} 23 .{ }^{22} \mathrm{MiR}-335-5 \mathrm{p}$ restrains thyroid cancer cell metastasis and invasion by targeting ICAM- $1 .{ }^{23} \mathrm{MiR}-$ 422a is crucial in cancer development ${ }^{24,25}$ and is involved in thyroid cancer modulation by interacting with IncRNAs and targeted genes. ${ }^{26,27}$ Besides, epigenetic regulators are involved in tumorigenesis and play critical roles in cancer development. ${ }^{28}$ Enhancer of zeste homolog 2 (EZH2), a histone methyltransferase, and sirtuin 6 (Sirt6), a deacetylase, are well-studied epigenetic regulators exhibiting essential roles in thyroid cancer development and targets of miRNAs. ${ }^{29,30}$ In the present study, we identified a novel function of $\mathrm{CCDC} 26$ in promoting thyroid cancer progression by regulating the miR-422a/EZH2/Sirt6 axis in vitro and in vivo.

\section{Materials and Methods}

\section{Clinical Thyroid Cancer Samples}

A total of 50 clinical thyroid cancer samples used in the study was obtained between August 2017 and August 2019 from Peking University Cancer Hospital and Institute. All patients were diagnosed and reviewed independently by two clinicians based on histopathological data as advanced thyroid carcinoma involving the anterior superior mediastinum and did not receive any systemic or local therapy before surgery. Among them, 32 had papillary adenocarcinoma, 6 had follicular adenocarcinoma, 6 had medullary carcinoma, and 4 had poorly differentiated carcinoma. The thyroid cancer tissues and corresponding para-neoplastic tissues obtained from the patients were snap-frozen in liquid nitrogen and stored at $-80^{\circ} \mathrm{C}$ before further analysis. All patients provided informed consent, and this study was approved by the Ethics Committee of Peking University Cancer Hospital and Institute (No. 76HG5573). This study conformed to the experimental guidelines of the World Medical Association and the Ethics Committee of Peking University Cancer Hospital and Institute.

\section{Cell Culture and Treatment}

Normal human thyroid Nthy-ori 3-1 cell line and thyroid cancer cell lines including FTC-133, SW579, TPC-1, and 8505C were purchased from Type Culture Collection of the Chinese Academy of Sciences (Shanghai) and cultured in DMEM (Solarbio, China) containing 10\% fetal bovine serum (Gibco, USA), $0.1 \mathrm{mg} / \mathrm{mL}$ streptomycin (Solarbio, China) and 100 units $/ \mathrm{mL}$ penicillin (Solarbio, China) at 37 ${ }^{\circ} \mathrm{C}$ with $5 \% \mathrm{CO}_{2}$. CCDC26 siRNA, CCDC26 shRNA, pcDNA3.1-CCDC26 overexpression vector, miR-422a mimic, miR-422a inhibitor, and corresponding controls were obtained from GenePharma (Shanghai, China). Cell transfection was performed by Lipofectamine 2000 (Invitrogen, USA) according to the manufacturer's instructions.

\section{Quantitative Reverse Transcription-PCR (qRT-PCR)}

Total RNAs were extracted by TRIZOL (Biosntech, China). The first-strand cDNA was synthesized following the manufacturer's instructions (Thermo, USA). qRT-PCR was carried out by applying SYBR-Green (Takara Biotechnology, Co., Lt., China). The standard control for miRNA and mRNA/lncRNA was U6 and GAPDH, respectively. Quantitative determination of RNA levels was conducted in three independent experiments. The primer sequences are as follows:

CCDC26 forward: 5'-CAAAGCTGGTCCTGTGCTTG -3 ; $\quad$ CCDC26 reverse: 5'-TTGAGTGTGGCA 
TCACTTCC-3'; miR-422a forward: 5'-GGCCTGGCTGG ACAGA-3'; miR-422a reverse: 5'-GTCA CATGACAACC CAGCTT-3'; EZH2 forward: 5'-TTGCCTGGATGATG GGGTCT-3'; EZH2 reverse: 5'-CATCTTAGTGGC CAGGTACA-3'; Sirt6 forward: 5'-GCCTTCTTCAGCCTGG AATAC-3'; Sirt6 reverse: 5'-TTCTGGAGTTCCTTG GTGGAGTG-3'; GAPDH forward: 5'-GGGCTGCTT TTAACT CTGGT-3'; GAPDH reverse: 5'-GCAGG TTTTTCTAGACGG-3'; U6 forward: 5'-CTCGCTTCGG CAGCACATATACT-3'; and U6 reverse: 5'-ACGC TTCACGAATTTGCGTGTC-3'.

\section{CCK-8 Assay}

Cell viability was analyzed by the CCK- 8 assays. About $5 \times 10^{3}$ cells were put into 96 wells and cultured for 12 hours and used for transfection or treatment. After 0 hours, 24 hours, 48 hours, 72 hours, and 96 hours, cells were incubated with a CCK-8 solution (KeyGEN Biotech, China) and cultured for another 2 hours at $37^{\circ} \mathrm{C}$. The absorbance at 450nm was measured with an ELISA browser (Bio-Tek EL 800, USA). ${ }^{31}$

\section{BrdU Incorporation Assay}

Cell proliferation was assessed by the BrdU incorporation assays. About $2 \times 10^{3}$ cells were put into 96 wells and cultured for 12 hours and used for transfection or treatment. After 0 hours, 24 hours, 48 hours, 72 hours, and 96 hours, cell proliferation was measured by BrdU Cell Proliferation Assay Kit (Yilaisa, China). ${ }^{32}$

\section{Colony Formation Assay}

About $1 \times 10^{3}$ SW579 and TPC-1 cells were layered in 6 wells and incubated in DMEM at $37^{\circ} \mathrm{C}$. After two weeks, cells were cleaned with PBS Buffer, fixed in methanol for thirty minutes, and stained with crystal violet dye at the dose of $1 \%$, after which the number of colonies was calculated. $^{31}$

\section{Transwell Assays}

Transwell assays were applied to analyze thyroid cancer cell invasion and migration using a Transwell plate (Corning, USA) according to the manufacturer's instruction. Briefly, the upper chambers were plated with around $1 \times 10^{5}$ cells and cultured for 48 hours. The migrated and invaded cells were solidified with $4 \%$ paraformaldehyde, stained with crystal violet, recorded and calculated. ${ }^{31}$

\section{Cell Apoptosis Analysis}

Around $2 \times 10^{5}$ cells were plated on 6 -well plates. Cell apoptosis was analyzed by using the Annexin V-FITC Apoptosis Detection Kit (CST, USA) according to the manufacturer's instruction. Briefly, about $2 \times 10^{6}$ collected and washed cells were resuspended in binding buffer and dyed at $25{ }^{\circ} \mathrm{C}$, followed by the flow cytometry analysis. ${ }^{33}$

\section{Luciferase Reporter Gene Assay}

Luciferase reporter gene assays were performed by using the Dual-luciferase Reporter Assay System (Promega, USA). Briefly, cells were treated with the miR-422a mimic, or control mimic, the vector containing CCDC26, CCDC26 mutant, EZH2, EZH2 mutant, Sirt6, and Sirt6 mutant fragment were transfected into the cells by using Lipofectamine 2000 (Invitrogen, USA), followed by the analysis of luciferase activities, in which Renilla was applied as a normalized control. ${ }^{31}$

\section{RNA Immunoprecipitation}

RNA immunoprecipitation (RIP) assays were conducted by an EZMagna RIP kit (Millipore, USA). Cells were lysed using a complete RIP lysis buffer. Cell extracts were incubated with magnetic beads conjugated to anti-AGO2 (Abcam, USA) or anti-IgG (Abcam, USA) antibodies for 6 hours, protein beads were removed, and RNA was purified and followed by qPCR analysis. ${ }^{34}$

\section{Western Blot Analysis}

Total proteins were extracted from the cells or mice tissues with RIPA buffer (CST, USA). Protein concentrations were measured by using the BCA Protein Quantification Kit (Abbkine, USA). The same amount of proteins were separated by SDS-PAGE (12\% polyacrylamide gels) and transferred onto PVDF membranes (Millipore, USA). The membranes were blocked with $5 \%$ milk and incubated overnight at $4^{\circ} \mathrm{C}$ with the primary antibodies for $\mathrm{EZH} 2$ (1:1000) (Amyjet, China), Sirt6 (1:1000) (Amyjet, China), and GAPDH (1:1000) (Amyjet, China), in which GAPDH served as the control. The corresponding second antibodies (1:1000) (Amyjet, China) were used for hatching the membranes for 1 hour at room temperature, followed by the visualization by using an Odyssey CLx Infrared Imaging System. 


\section{Tumorigenicity Analysis in Nude Mice}

In vivo tumor growth was analyzed in nude Balb/c mice (male, 4-week-old) that were randomly separated into four groups $(\mathrm{n}=4)$. To establish an in vivo tumor model, SW579 cells were treated with control shRNA (sh-NC), CCDC26 shRNA (sh-CCDC26), miR-422a inhibitor, or co-treated with CCDC26 shRNA (sh-CCDC26) and miR-422a inhibitor, respectively. Then, about $1 \times 10^{7}$ cells were subcutaneously injected into the mice. Tumor growth were measured every 7 days. After 35 days of injection, mice were sacrificed, and tumors were scaled. Tumor volume (V) was observed by measuring the length and width with calipers and calculated as measured with the method $\times 0.5$. Ki-67 expression in tumor tissues was tested by immunohistochemical staining with the Ki67 antibody (Santa Cruz Biotechnology, USA). EZH2, Sirt6, and GAPDH protein levels in the tumor tissues were analyzed by Western blot using primer antibodies against EZH2 (1:1000) (Amyjet, China), Sirt6 (1:1000) (Amyjet, China), and GAPDH (1:1000) (Amyjet, China), respectively. All animal handling procedures were approved by Peking University Cancer Hospital and Institute Animal Welfare Committee (No. 76HG5573) and complied with the authorized Animal Care and Method Procedure published by this hospital and the World Medical Association.

\section{Statistical Methods}

Data were presented as mean $\pm \mathrm{SD}$, and the statistical analysis was performed by SPSS 19.0 statistical software. The unpaired Student's $t$-test was applied for comparing two groups, and the one-way ANOVA was applied for comparing multiple groups. LSD test was used for subsequent analysis. $P<0.05$ were considered statistically significant.

\section{Results}

\section{CCDC26 is Upregulated in Thyroid}

\section{Cancer Tissues and Cell Lines}

To assess the potential correlation of CCDC26 with thyroid cancer progression, we analyzed its expression in the thyroid cancer tissues and cell lines. Significantly, our data showed that CCDC26 expression was elevated in thyroid cancer tissues $(\mathrm{n}=50)$ compared with the adjacent normal tissues $(\mathrm{n}=50)(P<0.01$, (Figure $1 \mathrm{~A}$ and $\mathrm{B})$, implying that $\mathrm{CCDC} 26$ is associated with thyroid cancer development. In addition, CCDC26 expression was upregulated in thyroid cancer cell lines, including FTC-133 $(P<0.01)$,
SW579 $(P<0.001)$, TPC-1 $(P<0.001)$, and 8505C $(P<$ $0.01)$ compared with the normal human thyroid Nthy-ori 3-1 cells (Figure 1C). SW579 and TPC-1 cells were selected for subsequent investigations. Moreover, based on the mean $\mathrm{CCDC} 26$ expression, thyroid cancer tissues $(n=50)$ were assigned into two groups for correlation analysis between CCDC26 and thyroid cancer patients' prognosis. We observed that a high CCDC26 level was associated with poor overall survival (OS) $(P<0.01$, Figure 1D), suggesting that CCDC26 expression is negatively correlated with thyroid cancer patients' prognosis. Taken together, these data suggest that CCDC26 is upregulated in thyroid cancer tissues and cell lines.

\section{CCDC26 Depletion Inhibits Thyroid Cancer Progression in vitro}

We further explored the effect of CCDC26 on thyroid cancer progression in vitro. To this end, CCDC26 was depleted by siRNA in SW579, and TPC-1 cells, and the knockdown efficiency was validated by qPCR $(P<0.001$, Figure 2A). CCK-8 assays revealed that CCDC26 depletion remarkably reduced SW579 and TPC-1 cells' viability $(P<0.01$, Figure 2B). Similarly, BrdU incorporation assays demonstrated that SW579 and TPC-1 cell proliferation was inhibited by CCDC26 knockdown $(P<0.01$, Figure 2C). Colony formation assays showed that CCDC26 knockdown decreased colony number in the cells $(P<0.01$, Figure 2D). Furthermore, Transwell assays demonstrated that $\mathrm{CCDC} 26$ depletion impaired the invasion and migration of SW579 and TPC-1 cells $(P<0.01$, Figure 2E and F). Meanwhile, CCDC26 knockdown remarkably induced apoptosis of SW579 and TPC-1 cells $(P<0.01$, Figure $2 \mathrm{G})$. Together, these results indicate that $\mathrm{CCDC} 26$ promotes thyroid cancer progression in vitro.

\section{CCDC26 Serves as a Sponge of miR-422a in Thyroid Cancer Cells}

Next, we identified the potential interaction between CCDC26 and miR-422a in the bioinformatic analysis using the web tool StarBase v.2.0 (Figure 3A). The miR-422a mimic remarkably reduced the luciferase activities of CCDC26 but failed to affect the CCDC26 with mutated miR-422a-binding site in SW579 and TPC-1 cells $(P<$ 0.01 , Figure 3B). RNA immunoprecipitation (RIP) assays showed that $\mathrm{CCDC} 26$ and miR-422a preferentially interacted with Ago2, but not IgG, in the microribonucleoprotein complexes (miRNPs) of SW579 and 
A

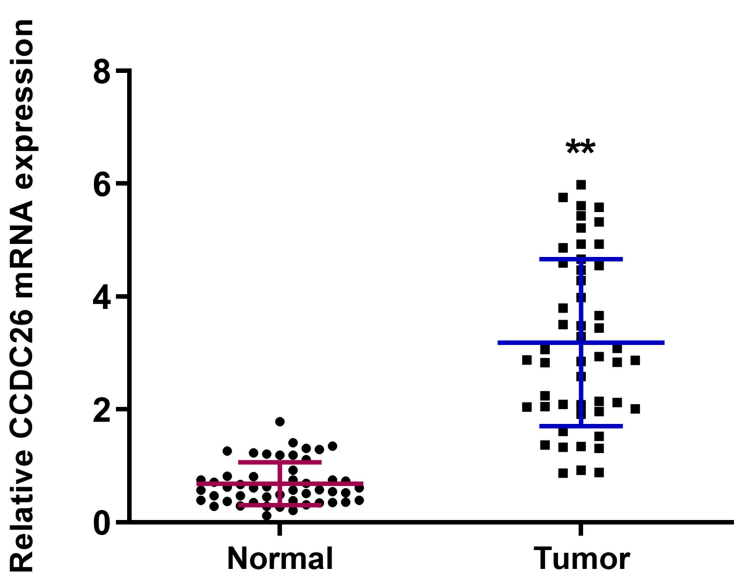

C

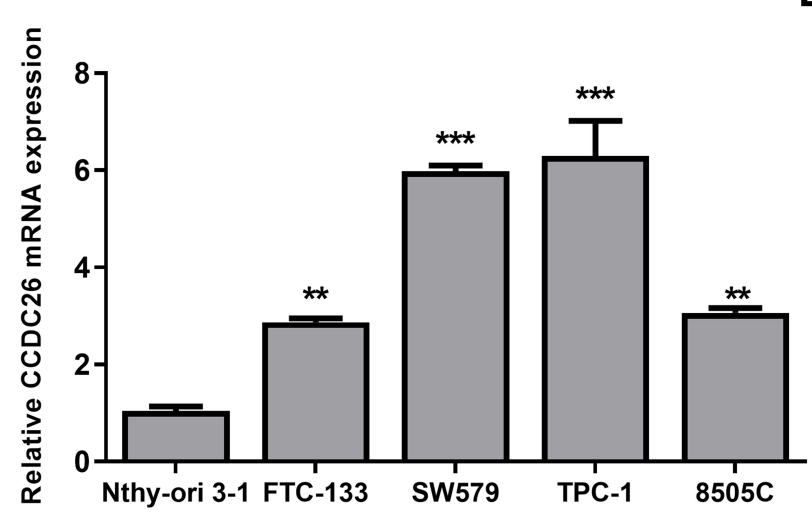

B

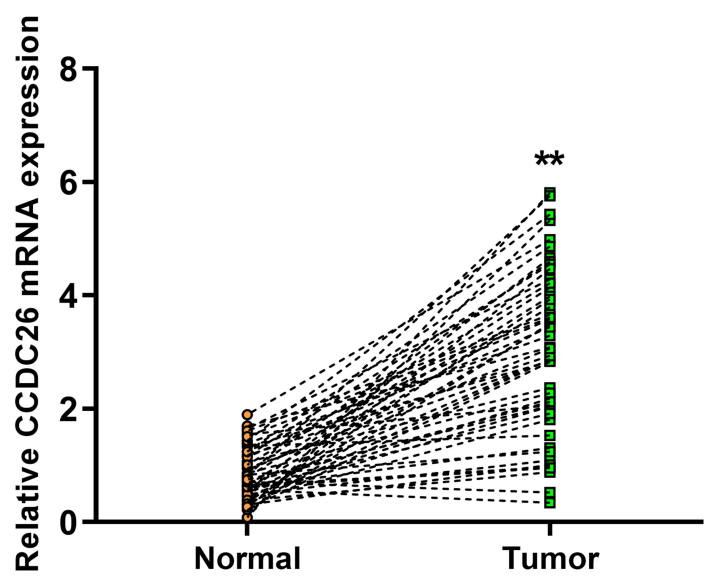

D

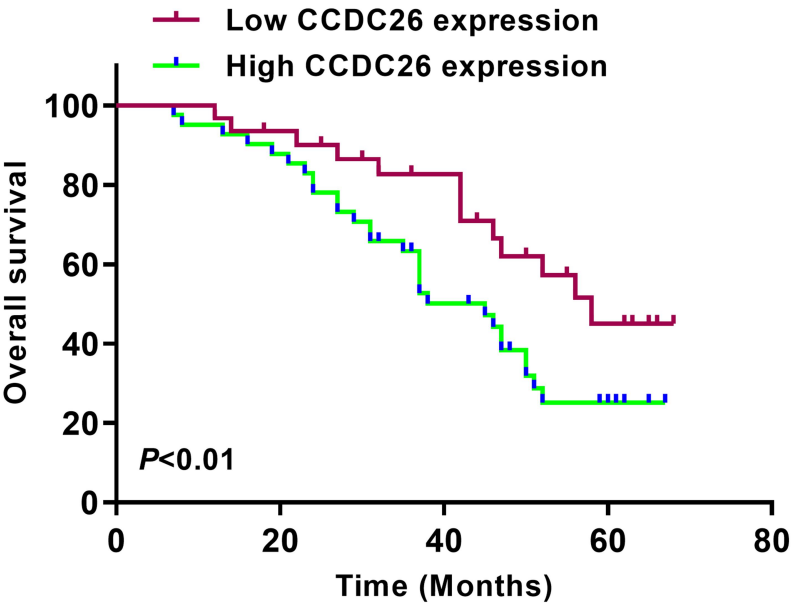

Figure I CCDC26 is upregulated in thyroid cancer tissues and cell lines. ( $\mathbf{A}$ and $\mathbf{B}$ ) CCDC26 expression measured by qPCR in thyroid cancer tissues ( $\mathrm{n}=50$ ) and adjacent normal tissues $(n=50)$. (C) CCDC26 expression assessed by qPCR in Nthy-ori 3-I, FTC-133, SW579, TPC-I, and 8505C cells. (D) The correlation of CCDC26 expression with overall survival (OS) of thyroid cancer patients analyzed by Kaplan-Meier analysis. Data are presented as mean \pm SD. Statistic significant differences were indicated: $* * P<0.01$, *** $P<0.001$.

TPC-1 cells $(P<0.01$, Figure 3C). CCDC26 depletion enhanced miR-422a expression in these cells $(P<0.01$, Figure 3D). Moreover, miR-422a expression was reduced in the thyroid cancer tissues $(n=50)$ compared with the adjacent normal tissues $(\mathrm{n}=50)(P<0.01$, Figure $3 \mathrm{E})$, and CCDC26 expression was negatively correlated with miR$422 \mathrm{a}$ in thyroid cancer tissues $(\mathrm{n}=50)(P<0.01$, Figure $3 \mathrm{~F})$, indicating the potential interplay of CCDC26 and miR-422a in the thyroid cancer progression. Together, CCDC26 serves as a sponge of miR-422a in thyroid cancer cells.

\section{MiR-422a Inhibitor Reverses CCDC26 Knockdown-Induced Inhibition of} Thyroid Cancer Progression in vitro We then explored the role of CCDC26/miR-422a axis in thyroid cancer development in vitro. CCK-8 assays revealed that CCDC26 depletion reduced $(P<0.01)$ while miR-422a inhibitor enhanced $(P<0.01)$ the viability of SW579 and TPC-1 cells, and miR-422a inhibitor reversed the effect of CCDC26 knockdown in these two cells $(P<0.05$, Figure 4A). BrdU incorporation assays showed that miR-422a inhibitor reversed CCDC26 knockdown-inhibited cell proliferation $(P<$ 0.05 , Figure 4B) and colony numbers $(P<0.05$, Figure 4C) and increased CCDC26 knockdownattenuated invasion and migration of SW579 and TPC1 cells $(P<0.05$, Figure 4D and E). Besides, CCDC26 depletion-induced apoptosis was blocked by miR-422a inhibitor $(P<0.05$, Figure $4 \mathrm{~F})$. Together, these data suggest that miR-422a inhibitor reversed CCDC26 knockdown-induced inhibition of thyroid cancer progression in vitro. 
A

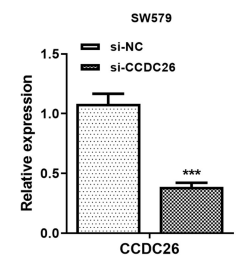

B

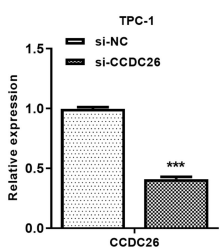

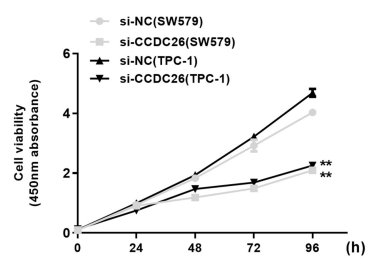

C

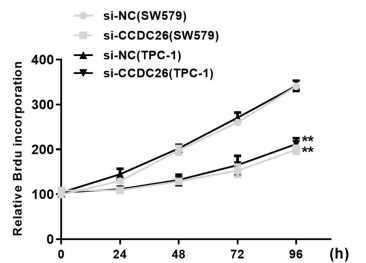

D

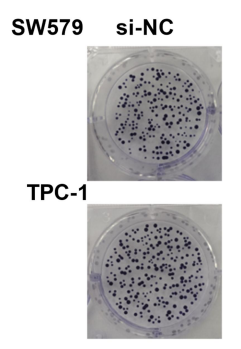

$\mathbf{F}$

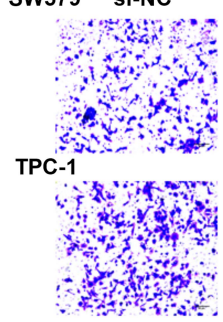

si-CCDC26

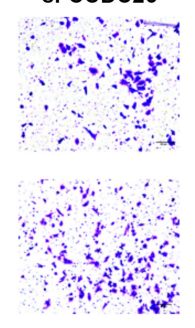

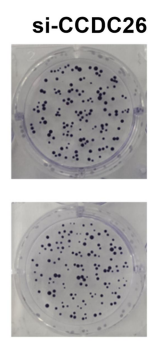

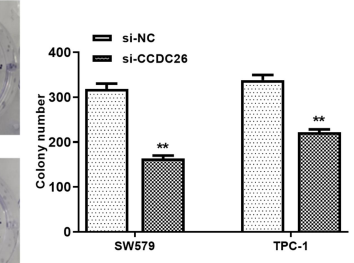

E
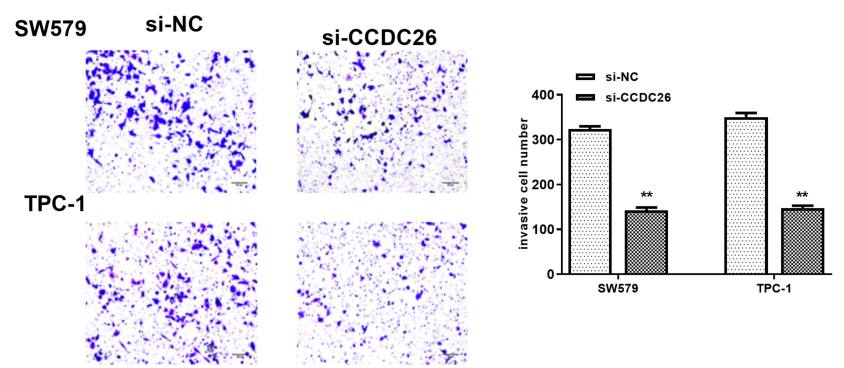

G
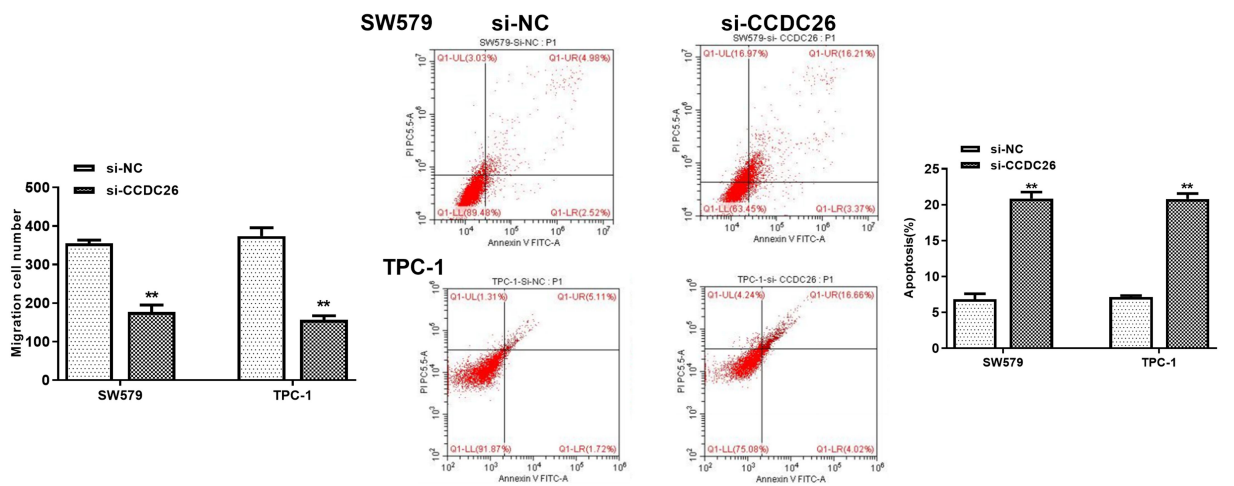

Figure 2 CCDC26 depletion inhibits thyroid cancer progression in vitro. (A-G) SW579 and TPC-I cells were transfected with control siRNA (si-NC) or CCDC26 siRNA (si-CCDC26). (A) CCDC26 expression measured by GPCR in these cells. (B) Viability of these cells analyzed by CCK-8 assays. (C and D) Proliferation of these cells assessed by BrdU incorporation assays and colony formation assays. (E and $\mathbf{F}$ ) Migration and invasion of these cells examined by Transwell assays. (G) Apoptosis of these cells measured by flow cytometry analysis. Data are presented as mean $\pm \mathrm{SD}$. Statistic significant differences were indicated: $* * P<0.01$, $* * * P<0.00 \mathrm{I}$.

\section{CCDC26 Upregulates EZH2/Sirt6 Expression by Sponging miR-422a in Thyroid Cancer Cells}

Next, we tried to identify miR-422a's target genes in thyroid cancer development. Bioinformatic analysis using Targetscan (http://www.targetscan.org/vert 72/) revealed miR-422a-targeted sites in EZH2 and Sirt6 3' UTR (Figure 5A). Notably, miR-422a mimic inhibited luciferase activities of wild type EZH2 and Sirt6 but failed to affect the EZH2 and Sirt6 with mutated miR-422a-binding sites in SW579 and TPC-1 cells $(P<0.01$, Figure 5B). miR422a mimic downregulated mRNA expression of EZH2 and Sirt6 $(P<0.01)$, and CCDC26 overexpression reversed this effect $(P<0.05$, Figure 5C). Besides, CCDC26 overexpression significantly rescued the protein expression of EZH2 and Sirt6 inhibited by miR-422a mimic in these cells $(P<0.05)$ (Figure 5D).
Furthermore, the expression of EZH2 and Sirt6 was increased in the thyroid cancer tissues $(\mathrm{n}=50)$ compared with the adjacent normal tissues $(\mathrm{n}=50)(P<0.01$, Figure $5 \mathrm{E})$, and miR-422a expression was negatively correlated with EZH2 and Sirt6 levels in thyroid cancer tissues $(\mathrm{n}=50)(P<0.01$, Figure 5F). Taken together, these results suggest that CCDC26 upregulates EZH2/Sirt6 expression by sponging miR-422a in thyroid cancer cells.

\section{CCDC26 Contributes to Thyroid Tumor Growth via miR-422a/EZH2/Sirt6 Axis \\ in vivo}

We further determined the impact of CCDC26/miR-422a/ EZH2/Sirt6 axis on thyroid cancer development in vivo. For this purpose, we performed tumorigenicity analysis in nude mice injected with SW579 cells, which were pretreated with control shRNA, CCDC26 shRNA, miR-422a 
A

miR-422a

CCDC26
ACUGGACUUAGGGUCAGAAGGC

:: :::::::::: ::::::

TGGCCTGAATCCCTGTCTTCTT
D

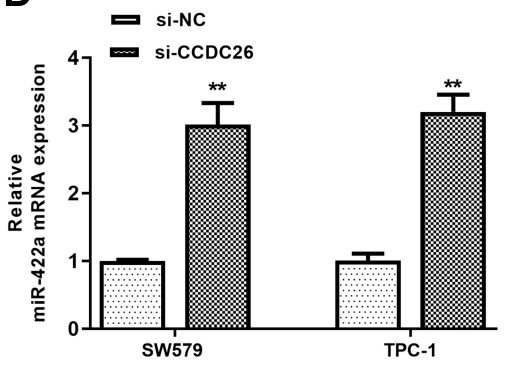

B

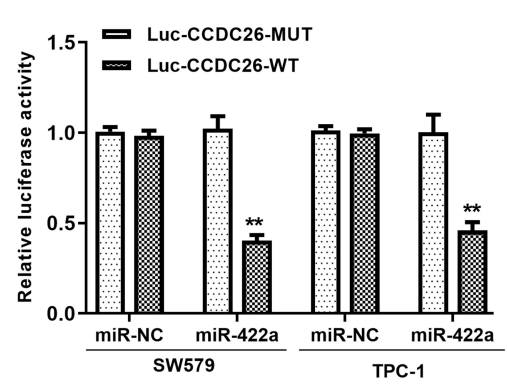

E

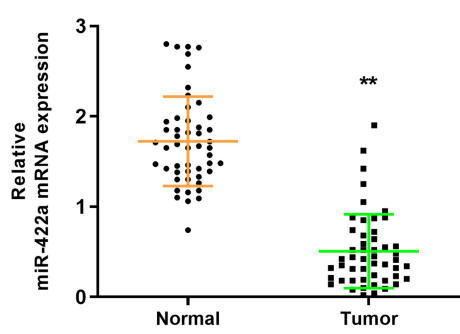

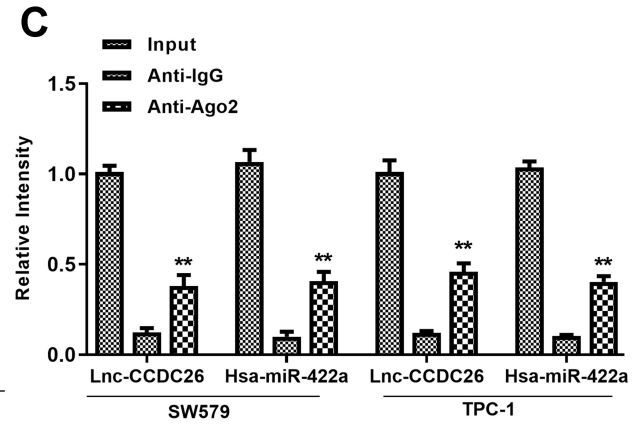

$\mathbf{F}$

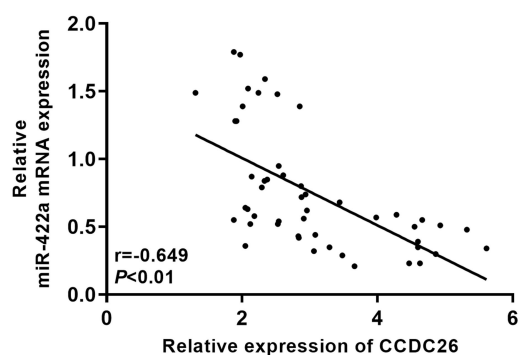

Figure 3 CCDC26 served as a sponge of miR-422a in thyroid cancer cells. (A) Potential interaction between CCDC26 and miR-422a identified by the bioinformatic analysis using the web tool StarBase v.2.0. (B) Luciferase activities in luciferase reporter gene assays in SW579 and TPC-I cells transfected with wild-type CCDC26 (CCDC26 WT), mutant CCDC26 with mutated miR-422a-binding site (CCDC26 MUT) and treated with control mimic (miR-NC) or miR-422a. (C) The interaction of CCDC26 and miR422a with Ago2 or IgG in SW579 and TPC-I cells examined by RNA immunoprecipitation (RIP) assays followed by qPCR. (D) MiR-422a expression measured by qPCR in the SW579 and TPC-I cells transfected with control siRNA (si-NC) or CCDC26 siRNA (si-CCDC26). (E) MiR-422a expression measured by qPCR in thyroid cancer tissues $(n=50)$ and adjacent normal tissues $(n=50)$. $(F)$ The correlation of CCDC26 with miR-422a analyzed by qPCR in thyroid cancer tissues ( $n=50)$. Data are presented as mean \pm SD. Statistic significant differences were indicated: $* * P<0.0$ l.

inhibitor, or co-treated with CCDC26 shRNA and miR422a inhibitor. Our data showed that CCDC26 depletion significantly reduced while miR-422a inhibitor enhanced the tumor growth in vivo, and miR-422a inhibitor reversed the effects of CCDC26 knockdown in the system, as demonstrated by the tumor size (Figure 6A), tumor weight $(P<0.05$, Figure $6 \mathrm{~B})$, tumor volume $(P<0.05$, Figure $6 \mathrm{C}$ ), and $\mathrm{Ki}-67$ expression in mouse tumor tissues (Figure 6D). Besides, we validated that CCDC26 knockdownattenuated expression of EZH2 and Sirt6 was enhanced by miR-422a inhibitors in mouse tumor tissues $(P<0.05$, Figure 6E). Together, these data suggest that $\mathrm{CCDC} 26$ contributes to thyroid tumor growth via miR-422a/EZH2/ Sirt6 axis in vivo.

\section{Discussion}

Thyroid cancer serves as a prevailing endocrine neoplasm globally with increasing mobility and severe morbidity. ${ }^{35}$ However, the mechanism of thyroid cancer progression remains unclear, extremely limiting the development of biomarkers for diagnosis, prognosis, and therapeutic candidates for thyroid cancer patients. ${ }^{36}$ In recent years, lncRNAs have been well-recognized to present critical roles in thyroid cancer pathogenesis. This study identified that lncRNA CCDC26 promoted thyroid cancer malignant progression via miR-422a/EZH2/Sirt6 axis.

As a crucial regulator in cancer development, lncRNAs are recognized to participate in thyroid cancer modulation. However, the role of CCDC26 in thyroid cancer is poorly understood despite some studies have revealed the function of CCDC26 in the progression of other cancer models. It has been reported that $\mathrm{CCDC} 26$ downregulation promotes imatinib resistance by upregulating IGF-1R and c-KIT in gastrointestinal stromal cancers. ${ }^{37,38} \mathrm{CCDC} 26$ depletion inhibits glioma cell migration and growth by sponging miR-203. ${ }^{39} \mathrm{CCDC} 26$ regulates cell growth by modulating KIT expression in myeloid leukemia. ${ }^{40}$ Nevertheless, the role of CCDC26 in thyroid cancer is still unreported. In this study, we, for the first time, identified that CCDC26 expression was elevated thyroid cancer tissues and cell lines, CCDC26 depletion reduced cell proliferation, migration, and invasion and induced apoptosis of thyroid cancer cells, and CCDC26 contributed to thyroid tumor growth in vivo. These data present a novel function of $\mathrm{CCDC} 26$ in promoting thyroid cancer progression, providing valuable evidence for the fundamental role 
A

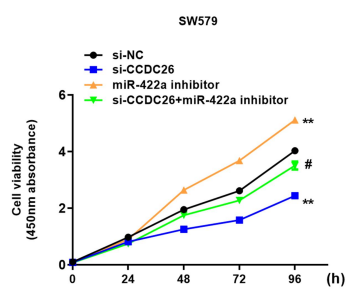

B

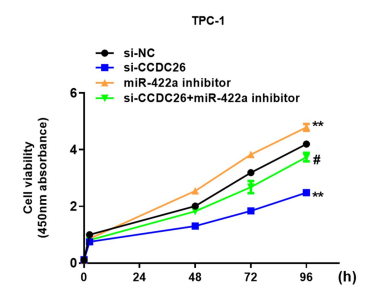

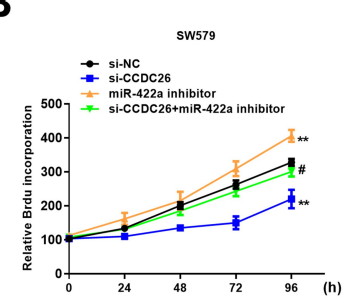
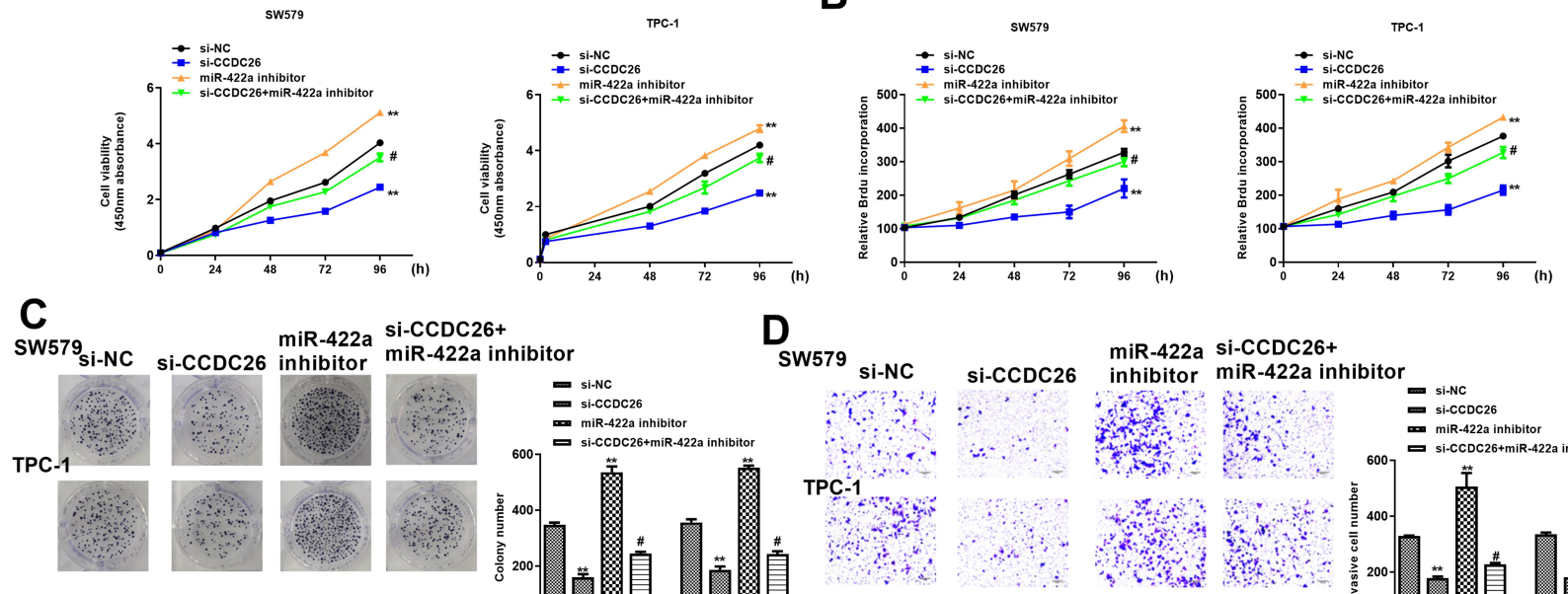

E

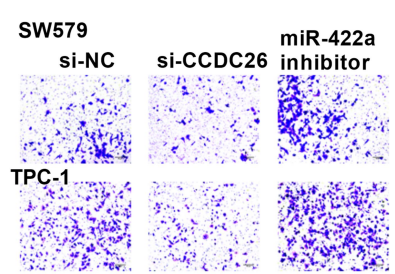

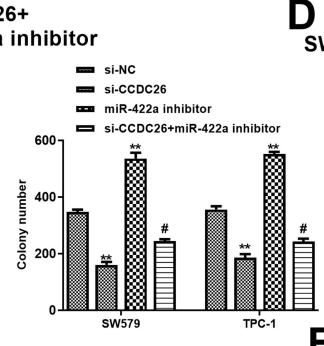

$F$ $\mathbf{D}_{\text {SW579 }}$ si-NC si-CCDC26 $\begin{aligned} & \text { miR-422a si-CCDC26+ } \\ & \text { inhibitor miR-422a inhibitor }\end{aligned}$

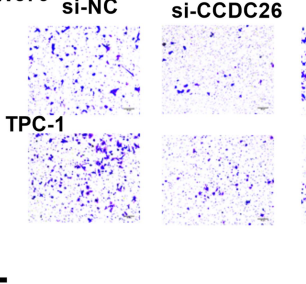

SW579 si-CCDC26+ mi-CCDC26+
miR-422a inhibitor

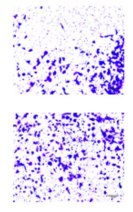

SW579 si-NC Si-CCDC26

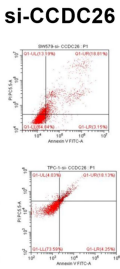

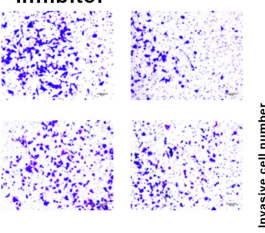

miR-422a si-CCDC26+
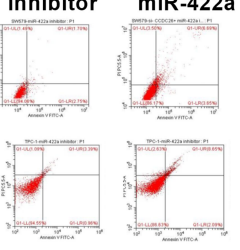

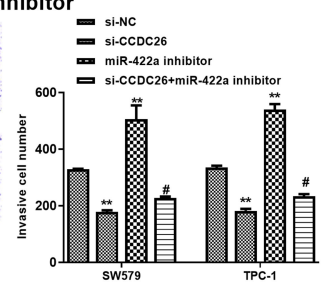
inhibitor miR-422a inhibitor

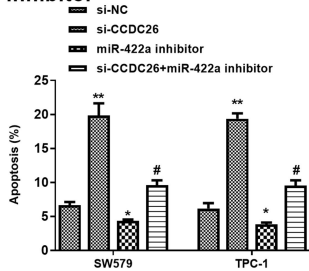

Figure 4 MiR-422a inhibitor reverses CCDC26 knockdown-induced inhibition of thyroid cancer progression in vitro. (A-F) The SW579 and TPC-I cells were transfected with control siRNA (si-NC), CCDC26 siRNA (si-CCDC26), miR-422a inhibitor, or co-treated with CCDC26 siRNA (si-CCDC26) and miR-422a inhibitor. (A) Cell viability analyzed by CCK-8 assays. (B) Cell proliferation measured by BrdU incorporation assays. (C) Cell proliferation assessed by colony formation assays. (D and E) Cell migration and invasion examined by Transwell assays. (F) Cell apoptosis measured by flow cytometry. Data are presented as mean \pm SD. Statistic significant differences were indicated: $* P<0.05, * * P<0.01,{ }^{\#} P<0.05$.

of lncRNAs in thyroid cancer development, which is consistent with the findings that a lncRNA can play similar roles in a variety of cancer models and how various lncRNAs work cooperatively in cancer development, although which needed to be further explored.

As a primary component of noncoding RNAs and a significant interplay factor with lncRNAs in the physiological and pathological processes, miRNAs are also involved in thyroid cancer modulation. It has been reported that miRNA-125b controls autophagy by interacting with Foxp3 in thyroid cancer. ${ }^{41}$ MiRNA-1270 regulates thyroid cancer progression through regulating SCAI. ${ }^{42}$ MiRNA-592 suppresses thyroid cancer development by downregulating NOVA1 and controlling lncRNA NEAT $1 .{ }^{43}$ MiRNA-15 mediates thyroid cancer invasion, migration, and proliferation by regulating Bcl-2. ${ }^{44}$ It has been identified that miR-422a participates in thyroid cancer modulation. miR-422a is involved in iodine/MAPK1-induced thyroid cancer progression. ${ }^{27}$ SP1-mediated lncRNA LINC00313 upregulation activates thyroid cancer tumorigenesis by modulating miR-422a. ${ }^{26}$ These investigations have well proven that miRNAs are regularly involved in the modulation of thyroid cancer progression. In the present study, we identified that miR-422a expression was reduced in thyroid cancer tissues. Our mechanism investigation further demonstrated that miR-422a inhibitor reversed CCDC26 knockdown-induced inhibition of thyroid cancer progression in vitro. These data displayed an unreported role of miR-422a in thyroid cancer development and identified a new association of miR-422a with CCDC26 in thyroid cancer modulation.

As two significant epigenetic regulators, EZH2 and Sirt6 have been well-identified to participate in the modulation of thyroid cancer development. It has been reported that miR-30d and miR-25 
A

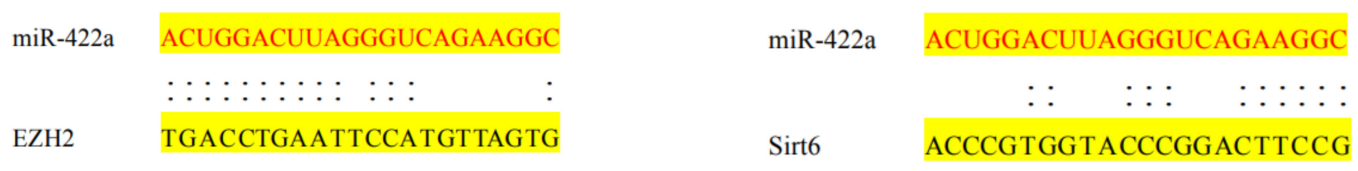

B

\section{C}
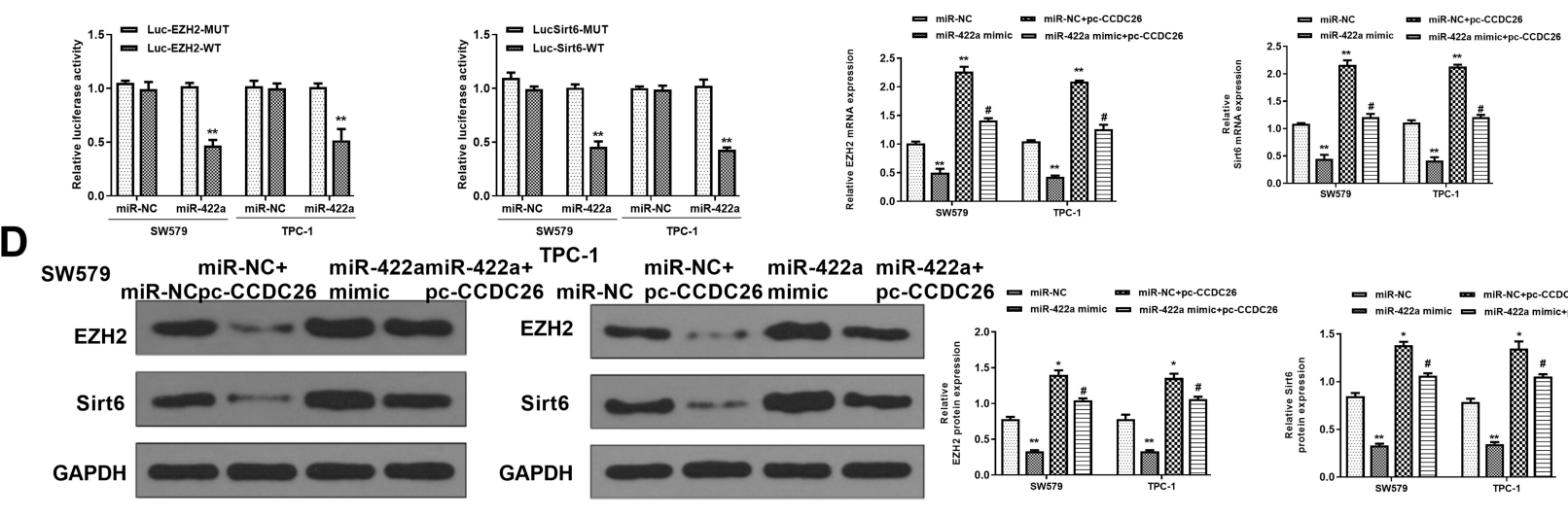

E
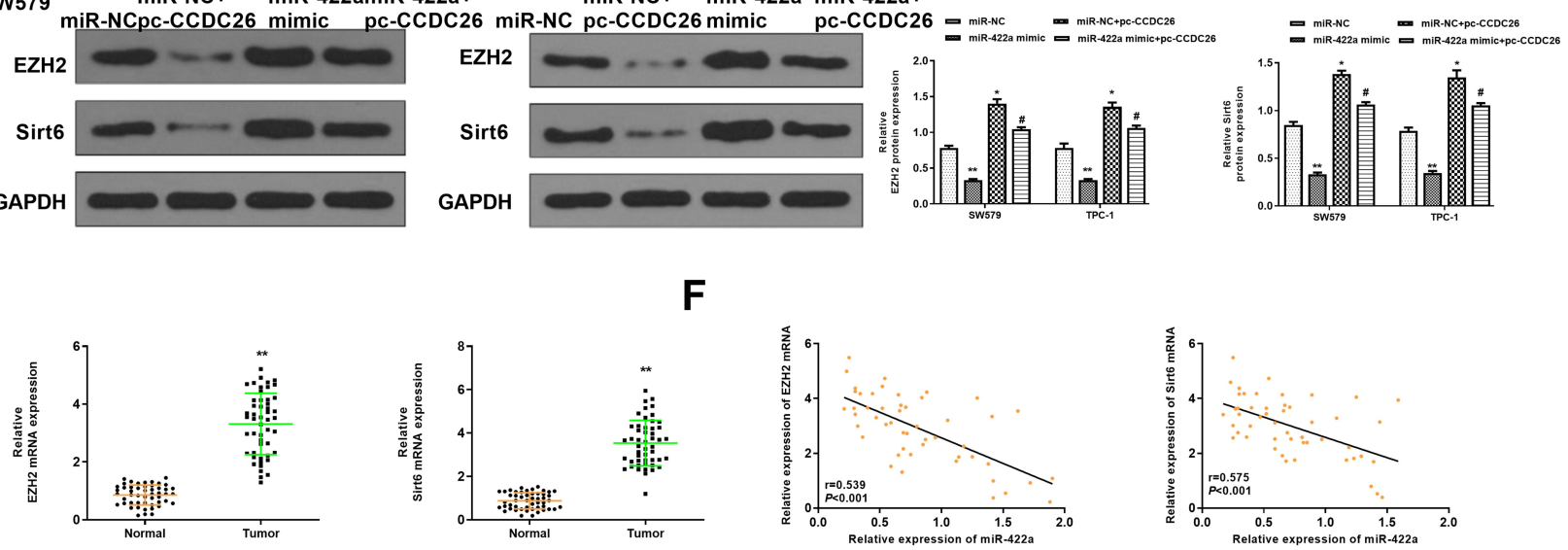

Figure 5 CCDC26 up-regulates EZH2/Sirt6 expression by sponging miR-422a in thyroid cancer cells. (A) The interaction of miR-422a with EZH2 and Sirt6 3' UTR identified by bioinformatic analysis using Targetscan (http://www.targetscan.org/vert_72/). (B) Luciferase activities in SW579 and TPC-I cells transfected with wild type EZH2 and Sirt6, and EZH2 and Sirt6 with mutated miR-422a-binding site determined by luciferase reporter gene assays. (C and D) SW579 and TPC-I cells were treated with control mimic (miR-NC), miR-422a mimic, or co-treated with pcDNA3.I-CCDC26 (pc-CCDC26) overexpression vector and control mimic (miR-NC) or miR-422a mimic. (C) EZH2 and Sirt6 mRNA levels measured by qPCR. (D) Protein expression of EZH2, Sirt6, and GAPDH tested by Western blot analysis. The results of Western blot analysis were quantified by Imagel software. (E) $E Z H 2$ and Sirt6 expression measured by qPCR in the thyroid cancer tissues $(n=50)$ and adjacent normal tissues ( $=50)$. (F) The correlation of EZH2 and Sirt6 with miR-422a analyzed by qPCR in thyroid cancer tissues $(n=50)$. Data are presented as mean \pm SD. Statistic significant differences were indicated: $* P<0.05, * * P<0.01,{ }^{\#} P<0.05$.

downregulation promotes thyroid cancer progression by targeting EZH2. ${ }^{29}$ EZH2 upregulation by ER $\alpha$ enhances thyroid cancer cell migration and proliferation. $^{45}$ In addition, Sirt6 contributes to the Warburg effect by regulating BCPAP through reactive oxygen species in papillary thyroid cancer cells. ${ }^{30}$ Sirt6/HIF-1 $\alpha$ signaling enhances the progression of papillary thyroid carcinoma by stimulating epithelialmesenchymal transition. ${ }^{46}$ Sirt6 is upregulated and correlated with malignancy aggressiveness by BRAF/ ERK/Mcl 1 signaling in papillary thyroid cancer. ${ }^{47}$ Our data showed that miR-422a targets EZH2 and Sirt6 in thyroid cancer cells, and CCDC26 upregulated EZH2/Sirt6 expression by sponging miR-422a in modulating thyroid cancer progression. These data provide new evidence that EZH2 and Sirt6 serve as the crucial factors in thyroid cancer development and present an unreported correlation of EZH2 and Sirt6 with miR$422 \mathrm{a}$ and CCDC26 in tumorigenesis. The effect of miR-422a on other potential targets in thyroid cancer development will be investigated in the future.

In conclusion, we identified that CCDC26 contributes to thyroid cancer malignant progression via miR-422a/ EZH2/Sirt6 axis. Our finding provides new insights into the mechanism by which CCDC26 promotes thyroid cancer malignant development and improves our understanding of the association of lncRNA with thyroid cancer. CCDC26 and miR-422a might serve as potential targets for thyroid cancer treatment. 
A

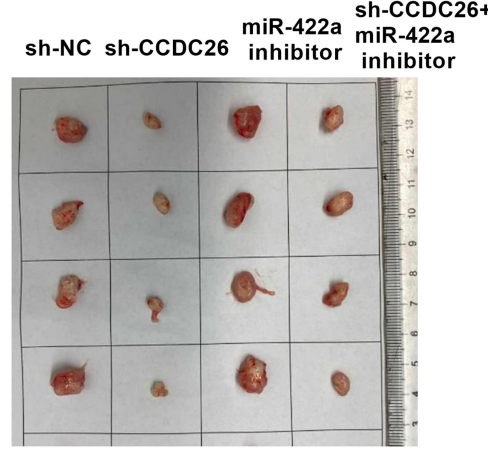

D

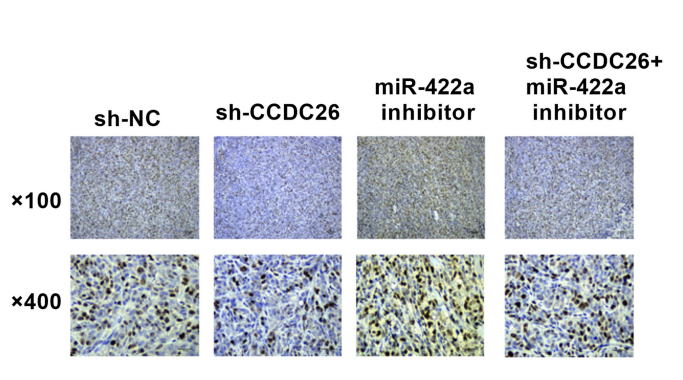

E
B

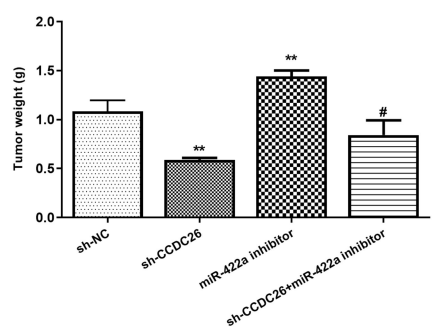

C

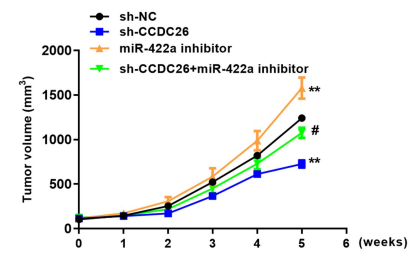

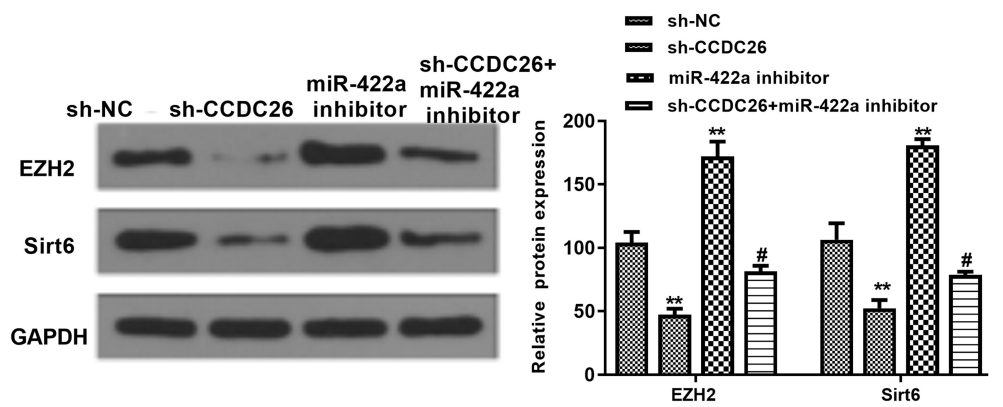

Figure 6 CCDC26 contributes to tumor growth of thyroid cancer via miR-422a/EZH2/Sirt6 axis in vivo. (A-E) The effect of CCDC26/miR-422a/EZH2/Sirt6 axis on tumor growth of thyroid cancer cells in vivo analyzed by nude mice tumorigenicity assay. SW579 cells were treated with control shRNA (sh-NC), CCDC26 shRNA (sh-CCDC26), miR-422a inhibitor, or co-treated with CCDC26 shRNA (sh-CCDC26) and miR-422a inhibitor and injected into the nude mice ( $\mathrm{n}=4$ ). (A) Representative images of dissected tumors from nude mice. (B) Average tumor weight. (C) Average tumor volume. (D) Ki-67 expression level in tumor tissues measured by immunohistochemical staining. (E) The protein expression levels of EZH2, Sirt6, and GAPDH in tumor tissues examined by Western blot analysis. The results of Western blot analysis were quantified by Imagej software. Data are presented as mean \pm SD. Statistic significant differences were indicated: $* * P<0.0$ I, ${ }^{\#} P<0.05$.

\section{Acknowledgments}

The authors would like to express our gratitude for those who have critically reviewed this manuscript and those who gave us help during this experiment.

\section{Funding}

This work was supported by the Science Foundation of Peking University Cancer Hospital (Grant No. 202003).

\section{Disclosure}

The authors report no conflicts of interest in this work.

\section{References}

1. Janjua N, Wreesmann VB. Aggressive differentiated thyroid cancer. Eur J Surg Oncol. 2018;44(3):367-377. doi:10.1016/j. ejso.2017.09.019

2. Sharifi A, Shojaeifard A, Soroush A, Jafari M, Abdehgah AG, Mahmoudzade H. Predictors of regional lymph node recurrence after initial thyroidectomy in patients with thyroid cancer. $J$ Thyroid Res. 2016;2016:4127278. doi:10.1155/2016/4127278

3. Wang Y, Wang W. Increasing incidence of thyroid cancer in Shanghai, China, 1983-2007. Asia Pac J Public Health. 2015;27(2):NP223-229. doi: $10.1177 / 1010539512436874$
4. Carlberg M, Hedendahl L, Ahonen M, Koppel T, Hardell L. Increasing incidence of thyroid cancer in the Nordic countries with main focus on Swedish data. BMC Cancer. 2016;16:426. doi:10.1186/s12885-016-2429-4

5. Xing M. Molecular pathogenesis and mechanisms of thyroid cancer. Nat Rev Cancer. 2013;13(3):184-199. doi:10.1038/nrc3431

6. Lundgren CI, Hall P, Dickman PW, Zedenius J. Clinically significant prognostic factors for differentiated thyroid carcinoma: a population-based, nested case-control study. Cancer. 2006;106 (3):524-531. doi:10.1002/cncr.21653

7. Schmidbauer B, Menhart K, Hellwig D, Grosse J. Differentiated thyroid cancer-treatment: state of the art. Int J Mol Sci. 2017;18(6). doi:10.3390/ijms18061292

8. Naoum GE, Morkos M, Kim B, Arafat W. Novel targeted therapies and immunotherapy for advanced thyroid cancers. Mol Cancer. 2018;17(1):51. doi:10.1186/s12943-018-0786-0

9. Peng WX, Koirala P, Mo YY. LncRNA-mediated regulation of cell signaling in cancer. Oncogene. 2017;36(41):5661-5667. doi:10.1038/ onc.2017.184

10. Kopp F, Mendell JT. Functional classification and experimental dissection of long noncoding RNAs. Cell. 2018;172(3):393-407. doi:10.1016/j.cell.2018.01.011

11. Bhan A, Soleimani M, Mandal SS. Long noncoding RNA and cancer: a new paradigm. Cancer Res. 2017;77(15):3965-3981. doi:10.1158/ 0008-5472.CAN-16-2634

12. Liu X, Fu Q, Li S, et al. LncRNA FOXD2-AS1 functions as a competing endogenous RNA to Regulate TERT expression by sponging miR-7-5p in thyroid cancer. Front Endocrinol (Lausanne). 2019;10:207. doi:10.3389/fendo.2019.00207 
13. Hou S, Lin Q, Guan F, Lin C. LncRNA TNRC6C-AS1 regulates $\mathrm{UNC5B}$ in thyroid cancer to influence cell proliferation, migration, and invasion as a competing endogenous RNA of miR-129-5p. J Cell Biochem. 2018;119(10):8304-8316. doi:10.1002/jcb.26868

14. Zheng W, Tian X, Cai L, et al. LncRNA DARS-AS1 regulates microRNA-129 to promote malignant progression of thyroid cancer. Eur Rev Med Pharmacol Sci. 2019;23(23):10443-10452. doi:10.26355/eurrev_201912_19683

15. Peng W, Jiang A. Long noncoding RNA CCDC26 as a potential predictor biomarker contributes to tumorigenesis in pancreatic cancer. Biomed Pharmacother. 2016;83:712-717. doi:10.1016/j. biopha.2016.06.059

16. Izadifard $\mathrm{M}$, Pashaiefar $\mathrm{H}$, Yaghmaie $\mathrm{M}$, et al. Expression analysis of PVT1, CCDC26, and CCAT1 long noncoding RNAs in acute myeloid leukemia patients. Genet Test Mol Biomarkers. 2018;22 (10):593-598. doi:10.1089/gtmb.2018.0143

17. Li D, Wang X, Lu S, et al. Integrated analysis revealing genome-wide chromosomal copy number variation in supraglottic laryngeal squamous cell carcinoma. Oncol Lett. 2020;20(2):1201-1212. doi: $10.3892 / 01.2020 .11653$

18. Lu TX, Rothenberg ME. MicroRNA. J Allergy Clin Immunol. 2018;141(4):1202-1207. doi:10.1016/j.jaci.2017.08.034

19. Rupaimoole R, Slack FJ. MicroRNA therapeutics: towards a new era for the management of cancer and other diseases. Nat Rev Drug Discov. 2017;16(3):203-222. doi:10.1038/nrd.2016.246

20. Zhou Q, Huang SX, Zhang F, et al. MicroRNAs: a novel potential biomarker for diagnosis and therapy in patients with non-small cell lung cancer. Cell Prolif. 2017;50(6). doi:10.1111/cpr.12394.

21. Ghafouri-Fard S, Shirvani-Farsani Z, Taheri M. The role of microRNAs in the pathogenesis of thyroid cancer. Noncoding RNA Res. 2020;5(3):88-98. doi:10.1016/j.ncrna.2020.06.001

22. Wen HL, Xu ZM, Lin SY, Wen D, Xie JP. miR-597-3p inhibits invasion and migration of thyroid carcinoma SW579 cell by targeting RAB23. Endokrynol Pol. 2020. doi:10.5603/EP.a2020.0041

23. Luo L, Xia L, Zha B, et al. miR-335-5p targeting ICAM-1 inhibits invasion and metastasis of thyroid cancer cells. Biomed Pharmacother. 2018;106:983-990. doi:10.1016/j.biopha.2018.07.046

24. Li WQ, Zhang JP, Wang YY, Li XZ, Sun L. MicroRNA-422a functions as a tumor suppressor in non-small cell lung cancer through SULF2-mediated TGF-beta/SMAD signaling pathway. Cell Cycle. 2019;18(15):1727-1744. doi:10.1080/15384101.2019.1632135

25. Zhang X, Gao D, Fang K, Guo Z, Li L. Med19 is targeted by miR-101-3p/miR-422a and promotes breast cancer progression by regulating the EGFR/MEK/ERK signaling pathway. Cancer Lett. 2019;444:105-115. doi:10.1016/j.canlet.2018.12.008

26. Yan DG, Liu N, Chao M, Tu YY, Liu WS. SP1-induced upregulation of long noncoding RNA LINC00313 contributes to papillary thyroid cancer progression via the miR-422a. Eur Rev Med Pharmacol Sci. 2019;23(3):1134-1144. doi:10.26355/eurrev_201902_17004

27. Wang J, Yang H, Si Y, et al. Iodine promotes tumorigenesis of thyroid cancer by suppressing Mir-422a and up-regulating MAPK1. Cell Physiol Biochem. 2017;43(4):1325-1336. doi:10.1159/000481844

28. Chen Y, Hong T, Wang S, Mo J, Tian T, Zhou X. Epigenetic modification of nucleic acids: from basic studies to medical applications. Chem Soc Rev. 2017;46(10):2844-2872. doi:10.1039/ C6CS00599C

29. Esposito F, Tornincasa M, Pallante P, et al. Down-regulation of the miR-25 and miR-30d contributes to the development of anaplastic thyroid carcinoma targeting the polycomb protein EZH2. J Clin Endocrinol Metab. 2012;97(5):E710-718. doi:10.1210/jc.2011-3068

30. Yu W, Yang Z, Huang R, Min Z, Ye M. SIRT6 promotes the Warburg effect of papillary thyroid cancer cell BCPAP through reactive oxygen species. Onco Targets Ther. 2019;12:2861-2868. doi:10.2147/ OTT.S194256
31. Zhao W, Han T, Li B, Ma Q, Yang P, Li H. miR-552 promotes ovarian cancer progression by regulating PTEN pathway. $J$ Ovarian Res. 2019;12(1):121. doi:10.1186/s13048-019-0589-y

32. Chen Y, Gao Y, Lian Y, Li C, Qu C, Jiang X. Overexpression of miR-221 stimulates proliferation of rat neural stem cell with activating Phosphatase and tensin homolog/protein kinase B signaling pathway. Neuroreport. 2020;31(14):1015-1023. doi:10.1097/ WNR.0000000000001513

33. Zou YT, Gao JY, Wang HL, Wang Y, Wang H, Li PL. Downregulation of microRNA-630 inhibits cell proliferation and invasion and enhances chemosensitivity in human ovarian carcinoma. Genet Mol Res. 2015;14(3):8766-8777. doi:10.4238/ 2015.July. 31.25

34. Kelly TJ, Suzuki HI, Zamudio JR, Suzuki M, Sharp PA. Sequestration of microRNA-mediated target repression by the Ago2-associated RNA-binding protein FAM120A. RNA. 2019;25 (10):1291-1297. doi:10.1261/rna.071621.119

35. Cabanillas ME, McFadden DG, Durante C. Thyroid cancer. Lancet. 2016;388(10061):2783-2795. doi:10.1016/S0140-6736(16)30172-6

36. Carling T, Udelsman R. Thyroid cancer. Annu Rev Med. 2014;65:125-137. doi:10.1146/annurev-med-061512-105739

37. Yan J, Chen D, Chen X, et al. Downregulation of IncRNA CCDC26 contributes to imatinib resistance in human gastrointestinal stromal tumors through IGF-1R upregulation. Braz J Med Biol Res. 2019;52 (6):e8399. doi:10.1590/1414-431x20198399

38. Cao K, Li M, Miao J, et al. CCDC26 knockdown enhances resistance of gastrointestinal stromal tumor cells to imatinib by interacting with c-KIT. Am J Transl Res. 2018;10(1):274-282.

39. Wang S, Hui Y, Li X, Jia Q. Silencing of lncRNA CCDC26 restrains the growth and migration of glioma cells in vitro and in vivo via targeting miR-203. Oncol Res. 2018;26(8):1143-1154. doi:10.3727/ $096504017 X 14965095236521$

40. Hirano T, Yoshikawa R, Harada H, Harada Y, Ishida A, Yamazaki T. Long noncoding RNA, CCDC26, controls myeloid leukemia cell growth through regulation of KIT expression. Mol Cancer. 2015;14:90. doi:10.1186/s12943-015-0364-7

41. Wang S, Wu J, Ren J, et al. MicroRNA-125b interacts with Foxp3 to induce autophagy in thyroid cancer. Mol Ther. 2018;26 (9):2295-2303. doi:10.1016/j.ymthe.2018.06.015

42. Yi T, Zhou X, Sang K, Zhou J, Ge L. MicroRNA-1270 modulates papillary thyroid cancer cell development by regulating SCAI. Biomed Pharmacother. 2019;109:2357-2364. doi:10.1016/j. biopha.2018.08.150

43. Luo Y, Hao T, Zhang J, Zhang M, Sun P, Wu L. MicroRNA-592 suppresses the malignant phenotypes of thyroid cancer by regulating IncRNA NEAT1 and downregulating NOVA1. Int $J$ Mol Med. 2019;44(3):1172-1182. doi:10.3892/ijmm.2019.4278

44. Lu Z, Wu Z, Hu J, Wei W, Ma B, Wen D. MicroRNA-15 regulates the proliferation, migration and invasion of thyroid cancer cells by targeting Bcl-2. J BUON. 2019;24(5):2114-2119.

45. Xue L, Yan H, Chen Y, et al. EZH2 upregulation by ERalpha induces proliferation and migration of papillary thyroid carcinoma. $B M C$ Cancer. 2019;19(1):1094. doi:10.1186/s12885-019-6306-9

46. Yang Z, Yu W, Huang R, Ye M, Min Z. SIRT6/HIF-1alpha axis promotes papillary thyroid cancer progression by inducing epithelial-mesenchymal transition. Cancer Cell Int. 2019;19:17. doi:10.1186/s12935-019-0730-4

47. Qu N, Hu JQ, Liu L, et al. SIRT6 is upregulated and associated with cancer aggressiveness in papillary thyroid cancer via BRAF/ERK/ Mcl1 pathway. Int J Oncol. 2017;50(5):1683-1692. doi:10.3892/ ijo.2017.3951 


\section{Publish your work in this journal}

OncoTargets and Therapy is an international, peer-reviewed, open access journal focusing on the pathological basis of all cancers, potential targets for therapy and treatment protocols employed to improve the management of cancer patients. The journal also focuses on the impact of management programs and new therapeutic

Submit your manuscript here: https://www.dovepress.com/oncotargets-and-therapy-journal agents and protocols on patient perspectives such as quality of life, adherence and satisfaction. The manuscript management system is completely online and includes a very quick and fair peer-review system, which is all easy to use. Visit http://www.dovepress.com/ testimonials.php to read real quotes from published authors. 